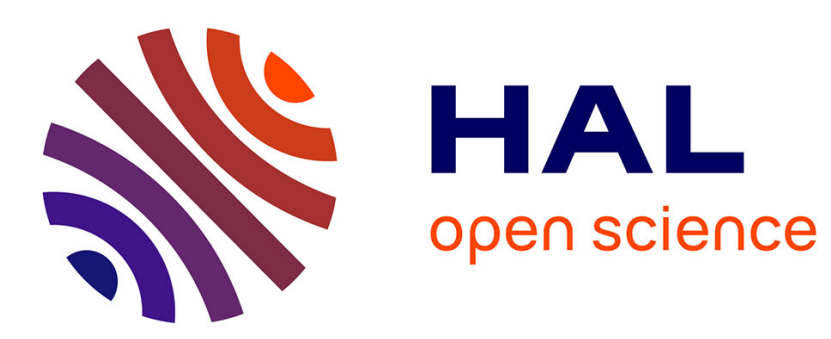

\title{
OXYDATION DE SiC SOUS OXYGĖNE ATOMIQUE ET MOLÉCULAIRE À HAUTE TEMPÉRATURE
}

\author{
M. Balat, G. Male, G. Flamant, B. Armas, M. Lebrun
}

\section{To cite this version:}

M. Balat, G. Male, G. Flamant, B. Armas, M. Lebrun. OXYDATION DE SiC SOUS OXYGÈnE ATOMIQUE ET MOLÉCULAIRE À HAUTE TEMPÉRATURE. Journal de Physique Colloques, 1990, 51 (C5), pp.C5-411-C5-417. 10.1051/jphyscol:1990549 . jpa-00230859

\section{HAL Id: jpa-00230859 https://hal.science/jpa-00230859}

Submitted on 1 Jan 1990

HAL is a multi-disciplinary open access archive for the deposit and dissemination of scientific research documents, whether they are published or not. The documents may come from teaching and research institutions in France or abroad, or from public or private research centers.
L'archive ouverte pluridisciplinaire HAL, est destinée au dépôt et à la diffusion de documents scientifiques de niveau recherche, publiés ou non, émanant des établissements d'enseignement et de recherche français ou étrangers, des laboratoires publics ou privés. 
COLLOQUE DE PHYSIQUE

Colloque C5, supplément au $\mathrm{n}^{\circ} 18$, Tome 51, 15 septembre 1990

OXYDATION DE SIC SOUS OXYGÈNE ATOMIQUE ET MOLECULAIRE À HAUTE TEMPERATURE

\author{
M. BALAT, G. MALE, G. FLAMANT, B. ARMAS et M. LEBRUN \\ Institut de science et de Génie des Matériaux et Procédés IMP-CNRS, UPR \\ 32. BP 5 Odeillo, 66120 Font-Romeu, France
}

\begin{abstract}
Résumé - Un plasma d'air généré par microondes est utilisé pour étudier 1'oxydation de matériaux massifs (Sic fritté) à basse pression $\left(\mathrm{P}_{02}^{\infty}\right.$ de 200 à $\left.2100 \mathrm{~Pa}\right)$, à haute température $(1673$ à $1973 \mathrm{~K})$ pour un débit d'air constant de $1,0610^{-6} \mathrm{~m}^{3} \cdot \mathrm{s}^{-1}$. Le comportement physico-chimique des échantillons placẻs au centre du plasma est comparé, dans les mêmes conditions expérimentales, à celui observé en atmosphère moléculaire. Des différences notables de comportement sont obtenues entre les deux atmosphères. La formation d'une couche de silice passivante a lieu sous oxygène atomique jusqu'à des pressions plus basses (pour la même température) que dans le cas de l'oxygène moléculaire.

Abstract - An air plasma generated by microwaves is used in order to study the oxidation of hot-pressed materials (SiC) at low pressure $\left(\mathrm{P}_{02}^{\infty}\right.$ from 200 to $\left.2100 \mathrm{~Pa}\right)$ and high temperature $\left(1673\right.$ to $1973 \mathrm{~K}$ ) for a constant air flow of $1.0610^{-6} \mathrm{~m}^{3} \mathrm{~s}^{-1}$. The physico-chemical behaviour of samples for the same experimental conditions is compared for two situations : plasma atmosphere and molecular atmosphere. Some significant differences are observed between the two atmospheres. The formation of $a$ "passive" layer of silica takes place under atomic oxygen until lower pressures (for the same temperature) than in the case of molecular oxygen.
\end{abstract}

\title{
1 - INTRODUCTION
}

Lors de la rentrée dans 1'atmosphère des véhicules spatiaux, l'onde de choc résultante produit des espèces excitées (ions, atomes, molécủles, électrons) qui conduisent à de fortes dégradations des matériaux de surface constituant la protection thermique de l'engin. A cette attaque de nature chimique se superpose l'échauffement de la protection qui reçoit des flux thermiques de l'ordre de 0,5 à 1 Mw. $\mathrm{m}^{-2}$, ceux-ci conduisant à des températures de surface maximales de l'ordre de $1500^{\circ} \mathrm{C}$ pour les bords d'attaque et de $1700^{\circ} \mathrm{C}$ pour le nez. Pour simuler partiellement ces conditions, nous avons réalisé au laboratoire un montage expérimental associant un moyen de chauffage de l'échantillon à haute température par rayonnement concentré et une chambre d'essai dans laquelle un plasma est produit.

\section{2 - MONTAGE EXPERIMENTAL}

La figure 1 représente l'ensemble du dispositif expérimental qui comprend :

- une enceinte cylinarique (1) de hauteur $500 \mathrm{~mm}$ et de diamètre intérieur $50 \mathrm{~mm}$ en silice (transparente au rayonnement solaire) munie à son extrémité supérieure d'une fenêtre en fluorine (2) permettant d'effectuer des visées pyrométriques. Ce tube traverse perpendiculairement le guide d'onde (3). Lorsque le plasma est formé, 
celui-ci occupe tout le volume autour de l'échantillon. Les expériences sont effectuées sous balayage d'air. Le débit est régulé par un débitmètre à bille (4) étalonné pour les différentes pressions de fonctionnement. A l'intérieur du réacteur, un tube en zircone muni d'un porte-échantilion en zircone (5) supporte les matériaux à traiter ;

- le générateur de microondes et le guide d'onde : nous avons utilisé un générateur de microondes (12) qui délivre une puissance réglable de 10 à $800 \mathrm{~W}$ à la fréquence de $2450 \mathrm{MHz}$. Nous avons au cours de l'ensemble des essais travaillé avec une puissance constante de $300 \%$. Un câble coaxial relie le gênérateur et le guide d'onde (3) muni de deux adaptateurs et du dispositif permettant de délimiter le plasma autour de l'échantillon. Le plasma d'air obtenu est de couleur rose avec une postdécharge jaune-vert caractéristique, la visualisation de l'accord est ainsi facile et réalisée avant toute insolation. La présence d'oxygène atomique a été vérifiée par spectrométrie d'émission. Le guide d'onde est rếfrigéré par une circulation d'eau à $1,510^{5} \mathrm{~Pa}$;

- le simulateur d'échauffement : tout le dispositif précédent (réacteur + guide d'onde) est placé au foyer d'un four solaire à concentration de rayonnement. Le flux incident est matérialisé sur la figure 1 par deux droites faisant un angle de $60^{\circ}$. Les flux disponibles sur ce moyen d'essai peuvent atteindre 4,5 MW. $\mathrm{m}^{-2}$, nous permettant ainsi d'obtenir facilement des températures élevées sur des matériaux de type Sic ;

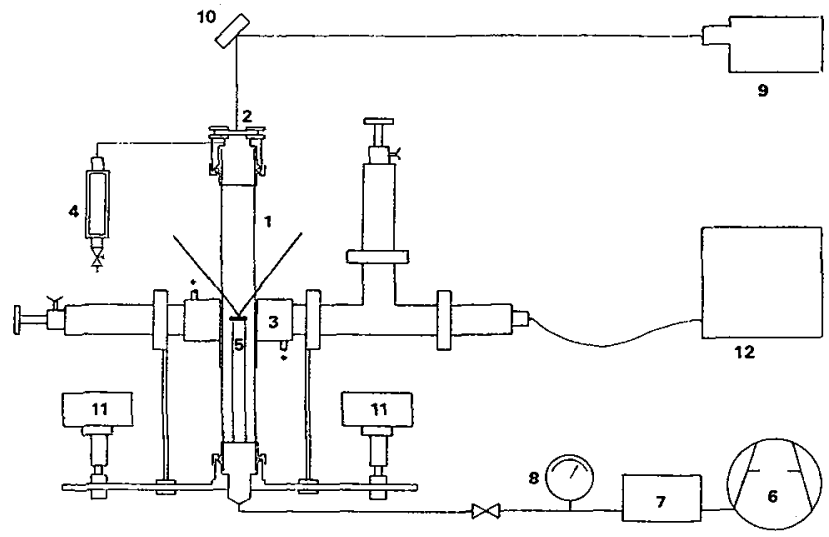

Figure 1 : Montage expérimental : (1) enceinte en silice, (2) fenêtre en fluorine, (3) guide d'onde, (4) débitmètre, (5) tube porte-échantillon, (6) pompe à vide, (7) régulateur de pression, (8) manomètre, (9) pyromètre optique, (10) miroir, (11) potence, (12) générateur microondes Experimental set-up : (1) silica vessel, (2) $\mathrm{CaF}_{2}$ window, (3) wave guide, (4) flowmeter, (5) sample-holder tube, (6) vacuum pump (7) pressure regulator, (8) pressure gauge, (9) optical pyrometer, (10) mirror, (11) support, (12) microwave generator.

- le système régulateur de pression: une pompe à palettes (6) $\mathrm{d}^{\prime}$ un débit maximal de $4,210^{-3} \mathrm{~m}^{3} \cdot \mathrm{s}^{-1}$ et un régulateur de pression (7) permettent un contrôle précis des conditions de pression au cours de l'expérimentation. Le suivi de la pression se fait grâce a un manomètre (8) ;

- le pyromètre : il s'agit d'un pyromètre Barnes (9) dont la longueur d'onde de mesure est centrée $\mathbf{a} ~ 5,5 \mu \mathrm{m}$, celle-ci nous permet d'effectuer des visées en face avant (= face insolée). Ce pyromètre délivre une tension $V$ proportionnelle à la température de couleur. La 
visée s'effectue par l'intermediaire d'un miroir (10) et d'une fenêtre en fluorine (2). L'etalonnage du pyrometre est effectué sur un corps noir Barnes dans les mêmes conditions (miroir + fenêtre et même distance entre source et pyromètre) que pour 1'expérience. La droite obtenue, dans le cas du corps noir, est $T\left({ }^{\circ} \mathrm{C}\right)=317.3+316.6 \mathrm{~V}(\mathrm{~V})$. La zone de mesure sur l'échantilion est un cerle de diamètre 7,6 m. La correction de la température par 1'émissivité est effectuée à postériori en prenant une valeur moyenne pour $\varepsilon(\lambda=5,5 \mu \mathrm{m})=0,90$ pour sic et $\mathrm{SiO}_{2} / 1 /$. L'erreur sur la température due a la correction d'émissivité est́ a $1500^{\circ} \mathrm{C}$ de \pm $45^{\circ}$ selon que $l^{\prime}$ on prend $\varepsilon=0,95$ ou $\varepsilon=0,85$, soit une précision de $3 \%$ à $1500^{\circ} \mathrm{C}$. Le tableau 1 résume les caractéristiques principales du montage experimental.

Tableau 1 : Principales caractéristiques du montage experimental.- Main characteristics of the experimental set-up.

\begin{tabular}{|c|c|c|c|}
\hline $\begin{array}{l}\text { Générateur } \\
\text { microondes }\end{array}$ & $\begin{array}{l}\text { Atmosphère } \\
\text { Pression }\end{array}$ & $\begin{array}{l}\text { Simulateur } \\
\text { d'échauffement }\end{array}$ & Echantillon \\
\hline $\begin{array}{l}2450 \mathrm{MHz} \\
\mathrm{P}_{\mathrm{max}}=800 \mathrm{~W} \\
\mathrm{P}_{\mathrm{trav}}=300 \mathrm{~W} \\
\text { (due au câble } \\
\text { coaxial) }\end{array}$ & $\begin{array}{l}\text { Air, } \mathrm{O}_{2} \text { etc } \\
\mathrm{P} \text { variable de } \\
50 \mathrm{a} 15 \cdot 10^{3} \mathrm{~Pa} \\
(1 \text { imite à } \\
\text { cause des } 300 \mathrm{w})\end{array}$ & $\begin{array}{c}\text { Flux max }=4.5 \mathrm{MW} \cdot \mathrm{m}^{-2} \\
\mathrm{~T} \max =2273 \mathrm{~K} \\
\text { vitesse montée ou } \\
\text { descente en T : } \\
\text { mini } 0.5 \% / \mathrm{s} \\
\text { maxi } 10 \% / \mathrm{s}\end{array}$ & $\begin{array}{l}\text { Diamètre }= \\
10 \text { à } 40 \mathrm{~mm}\end{array}$ \\
\hline
\end{tabular}

\section{3 - ETUDE THERMODYNAMIOUE THEORIOUE}

La disparité des données $/ 2,3,4 /$ concernant la transition entre l'oxydation "passive" (formation d'une couche de $\mathrm{SiO}_{2}$ passivante correspondant $\mathrm{a}$ un gain de masse de l'échantilion) et l'oxydation "active" (vaporisation de sio et $\mathrm{co}$, dans le cas de $\mathrm{sic}$, avec une perte de masse de l'échantillon) nous a conduit à faire avant toute expêrimentation un calcul thermodynamique pour déterminer la transition. Cette étude est évoquée brièvement ici et sera publiée prochainement.

Avant passivation par la couche de silice, l'interface solide/gaz est constituée de la couche de carbure de silicium solide et des phases vapeurs majoritaires $(\mathrm{SiO}),\left(\mathrm{O}_{2}\right),(\mathrm{CO})$ et $\left(\mathrm{N}_{2}\right)$. A pression et température fixées, le système est monovariant. Donc, pour une pression partielle d'oxygène fixée, le système est entièrement déterminé. Pour chaque phase, on effectue sur chaque espèce atomique, le bilan de matière puis on écrit la conservation à l'interface. En faisant les hypothèses suivantes (justifiées à posteriori) : pression partielle $d^{\prime}$ oxygène interfaciale $\mathrm{P}_{2}^{\mathrm{W}}$ très petite (consommation) et pression partielle de Sio dans le gaz vecteur $P_{S}^{\infty}{ }_{i o}$ voisine de zéro (hypothèse de la couche limite de concentration), on obtient finalement : $P_{02}^{\infty}=\left(D_{s_{i}} / D_{02}\right)^{1 / 2}$. $P_{s i 0}^{w}$ avec $D_{s i 0}$ et $D_{02}$ les coefficients de diffusion des espèces sio et $\mathrm{O}_{2}$ en phase gazeuse. On exprime ensuite la pression partielle d'oxygène dans le gaz vecteur $\mathrm{P}_{02}^{\infty}$ en fonction des paramètres thermodynamiques en faisant intervenir les deux équilibres suivants :

$$
\begin{aligned}
& \langle\mathrm{SiC}\rangle+\left(\mathrm{O}_{2}\right) \rightleftharpoons(\mathrm{SiO})+(\mathrm{CO})=\left\langle\mathrm{SiO}_{2}\right\rangle+(\mathrm{CO}) \\
& \langle\mathrm{SiC}\rangle+3 / 2\left(\mathrm{O}_{2}\right) \rightleftharpoons \Delta \mathrm{G}_{2}^{\circ}
\end{aligned}
$$

ceci étant valable à la transition puisque l'équilibre thermodynamique est réalisé. On a donc : 


$$
\begin{aligned}
& \mathrm{K}_{1}=\exp \left(-\Delta \mathrm{G}_{1}^{0} / \mathrm{RT}\right)=\mathrm{P}_{\mathrm{S} i 0}^{\mathrm{w}} \cdot \mathrm{P}_{\mathrm{C} 0}^{\mathrm{w}} / \mathrm{P}_{02}^{w} \\
& \mathrm{~K}_{2}=\exp \left(-\Delta \mathrm{G}_{2}^{0} / \mathrm{RT}\right)=\mathrm{P}_{\mathrm{C} 0}^{\mathrm{w}} \cdot\left(\mathrm{P}_{02}^{\mathrm{w}}\right)^{-3 / 2}
\end{aligned}
$$

En poursuivant le calcul, on obtient :

$$
\mathrm{P}_{\mathrm{S} i 0}^{\mathrm{w}}=\mathrm{K}_{1}^{3 / 4} \cdot \mathrm{K}_{2}^{-1 / 2}\left(\mathrm{D}_{\mathrm{S} \text { i } 0} / \mathrm{D}_{\mathrm{C} 0}\right)^{-1 / 8}
$$

Avec l'hypothèse de l'égalité des coefficients de diffusion $D_{c_{0}}=D_{02}$ (molécules de taille et de masse très voisines), il vient :

$$
P_{02}^{\infty}=K_{1}^{3 / 4} \cdot K_{2}^{-1 / 2}\left(D_{s i o} / D_{02}\right)^{3 / 8}
$$

Pour obtenir des valeurs numériques, nous avons utilisé les tables de JANAF /5/ et pour le rapport des diffusivités massiques la valeur donnée par WAGNER $/ 2 /$ de 0,64 . Quelques unes des valeurs obtenues sont données dans le tableau 2 .

Tableau 2 : Valeurs théoriques de la pression partielle d'oxygène en fonction de la température pour la transition.- Theoretical values of the oxygen partial pressure versus temperature for the transition.

\begin{tabular}{|l|c|c|c|c|c|c|c|}
\hline$T(K)$ & 1473 & 1573 & 1673 & 1773 & 1873 & 1973 & 2073 \\
\hline$P_{02}^{\infty}(\mathrm{Pa})$ & 10,3 & 65,5 & 342,5 & 1436 & 5077 & $15,7 \quad 10^{3}$ & $43,210^{3}$ \\
\hline
\end{tabular}

Par exemple, à $T=1673 \mathrm{~K}$, la théorie prévoit une transition pour $P_{02}^{\infty}=$ $342,5 \mathrm{~Pa}$ or d'après SINGHAL /4/ elle se produit à $103 \mathrm{~Pa}$ (données JANAF 1971) et d'après GULBRANSEN /3/ elle est comprise entre 32 et $582,6 \mathrm{~Pa}$, ceci impliquant donc la formation d'une couche protectrice pour des pressions partielles d'oxygène supérieures ou égales à celles-ci. Il faut signaler que, du point de vue thermodynamique, la prise en compte dans le calcul de $\left(\mathrm{O}_{2}\right)$ ou de $(0)$ conduit au même résultat quant à la transition oxydation active $\rightarrow->$ oxydation passive.

\section{4 - ETUDE EXPERIMENTALE}

Une fois les conditions de pression et de température déterminées pour la transition entre oxydation "passive" et "active", nous avons réalisé les expérimentations regroupées dans le tableau 3 .

\begin{tabular}{|c|c|c|c|c|c|}
\hline$\underbrace{\infty}_{02}(\mathrm{~Pa})$ & 1673 & 1773 & 1805 & 1870 & 1950 \\
\hline $\begin{array}{r}200 \\
400 \\
700 \\
1000 \\
2100\end{array}$ & 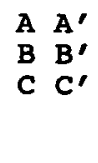 & ${ }_{E}^{D} D^{\prime}$ & $F^{\prime}$ & $G^{\prime}$ & $\mathbf{H}^{\prime}$ \\
\hline
\end{tabular}

Tableau 3 : Expériences réalisées sous $O_{2}$ (points A à E) et sous oxygène atomique (points $A^{\prime}$ à $H^{\prime}$ ). - Experiments done under $O_{2}$ (points $A$ to $E$ ) and atomic oxygen (points $A^{\prime}$ to $H^{\prime}$ ).

Les échantillons étudiés proviennent de "Céramiques et composites" (Rhône-Poulenc). Il s'agit de disques de sic fritté ( $\alpha$ sic-6H) dont la composition est la suivante : $98,5 \%$ volume de $\operatorname{sic}, 1 \%$ de $B,<2000$ ppm de 
Si et $\mathrm{SiO}_{2},<1000 \mathrm{ppm}$ de $\mathrm{Fe}, \mathrm{C}$ et $(\mathrm{Na}+\mathrm{K}+\mathrm{Ca}+\mathrm{Mg})$. Leur diamètre est de $25 \mathrm{~mm}$ et l'épaisseur $3 \mathrm{~mm}$ avec une des faces polies à $6 \mu \mathrm{m}$ (face sur laquelle on réalise les mesures optiques de température).

\section{4-1 = Protocole experimental}

Après positionnement de l'échantillon dans le réacteur en silice, le vide est réalisé dans l'ensemble de l'installation. Puis, les conditions de pression et débit sont établies et la pression stabilisée. Pour les expériences sous plasma, le plasma est mis en route après stabilisation de la pression et avant insolation de l'échantillon. Ensuite, l'exposition de l'échantilion au rayonnement est réalisée. Nous pouvons apprécier la concentration en atomes d'oxygène. D'après DIAMY $/ 6 \%$, pour les mêmes conditions de puissance microondes, de débit et de pression, le taux de dissociation est de 158 environ dans le plasma ce qui dans notre cas donne un flux atomique de $2.10^{15}$ at. $\mathrm{s}^{-1} . \mathrm{cm}^{-2}$. Grâce à l'ouverture progressive de l'obturateur du concentrateur, la montée en température $s^{\prime}$ effectue à une vitesse de $4^{\circ} \mathrm{s}^{-1}$ puis on maintient un palier de température pendant 360 ou $420 \mathrm{~s}$ selon les expériences et la descente s'effectue avec une vitesse de $2^{\circ} \mathrm{s}^{-1}$ comme en témoigne l'histoire thermique de la figure 2. La variation de masse de 1'échantillon s'effectue par pesée avant et après essai. La présence d'une couche de $\mathrm{SiO}_{2}$ est déterminée par pesée et par diffraction $\mathrm{x}$. Une etude de cette couche et de I'interface $\mathrm{SiC}^{-\mathrm{SiO}_{2}}$ est envisagée par spectroscopie Auger. Les points expérimentaux sont reportés sur la figure 3 comportant la droite théorique log $P_{02}^{\infty}=f(1 / T)$ correspondant à la transition oxydation active $\longleftrightarrow->$ oxydation passive.

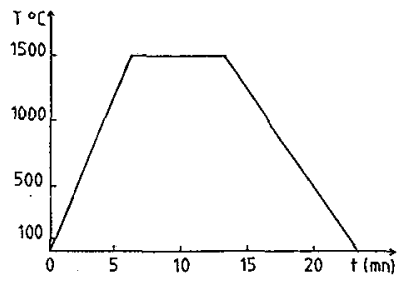

Figure 2 : Histoire thermique d'un echantillon.- Thermal history of a sample. $\log \mathrm{P}_{\mathrm{O}_{2}}^{\infty}$ (atm)

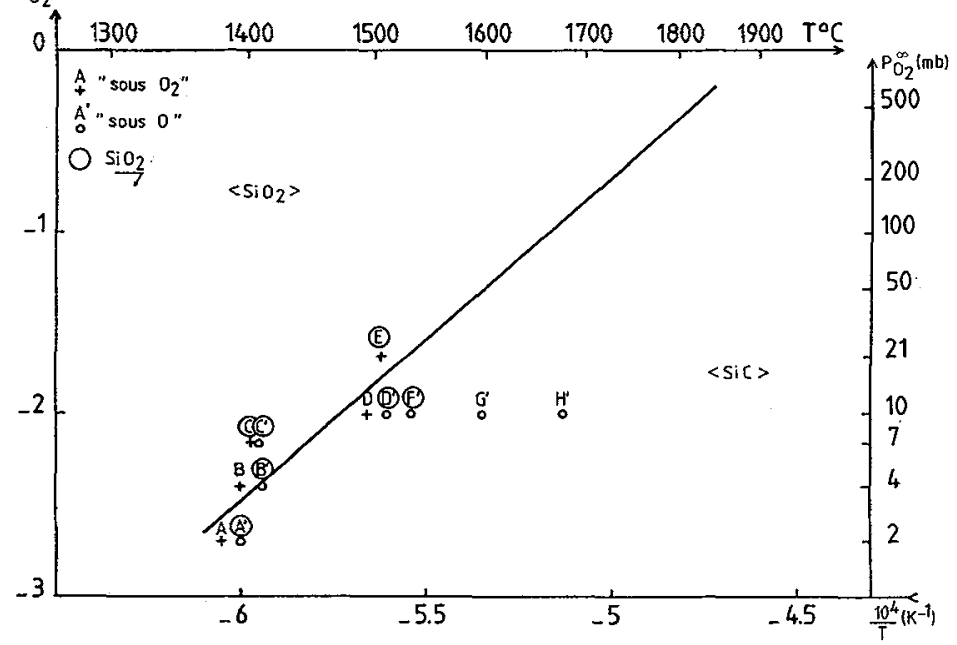

Figure 3 : Pression partielle d'oxygène en fonction de la température : droite théorique (calcul thermodynamique) et points expérimentaux (A+ 
sous $\mathrm{O}_{2}, A^{\prime} \mathrm{O}$ sous $\left.\mathrm{O}\right)$. Les lettres cerclées représentent les échantillons sur lesquels il $y$ a eu formation d'une couche de silice passivante.oxygen partial pressure versus temperature : theoretical straight 1ine (thermodynamic calculation) and experimental points (At under $\mathrm{O}_{2}, A^{\prime} \mathrm{O}$ under 0$)$. The letters surrounded with a circle represent the samples with a passive silica layer.

\section{$\underline{4-2}=$ Expérimentation sous oxygène moléculaire}

Pour valider la prévision thermodynamique, nous avons réalisé les expériences matérialisees par les points $A, B, C, D$ et E. Pour les échantillons, $A, B$ et $D$, à la suite de l'oxydation, la surface des échantillons est composée de Sic; il $y$ a donc eu pour ces 3 essais, vaporisation de sio et de $c o$ et donc oxydation active avec des pertes de masse respectives de $2,2,2$ et $3,6 \%$. Notons cependant que, compte tenu de la précision sur la mesure de température, le point $B$ qui est à $17^{\circ}$ de la droite théorique devrait se situer dans le domaine d'existence de Sic (les points $A, D$ et $E$ sont respectivement a 21,22 et $23^{\circ}$ de la droite). Pour les échantillons $C$ et $E$, la surface est recouverte par une couche de $\mathrm{SiO}_{2}$ passivante, très fine, la variation de poids entre le début et la fin de l'essai est positive mais négligeable. Ces différents essais sous oxygène moléculaixe ont permis de valider le calcul thermodynamique avec une bonne précision $(3 \%)$.

\section{$\underline{4-3}$ = Expérimentation sous oxygène atomique}

Pour les échantillons $A^{\prime}, B^{\prime}, C^{\prime}, D^{\prime}$ et $F^{\prime}, 1^{\prime}$ oxydation a été passivante car il y a eu formation d'une couche trés fine de $\mathrm{SiO}_{2}$. Pour les échantillons $G^{\prime}$ et $H^{\prime}$ dont les températures de surface au palier sont respectivement de 1870 et $1950 \mathrm{~K}$, il $\mathrm{Y}$ a eu vaporisation de sio et de co avec des pertes de masse respectives de 2,5 et $4 \%$. Dans le cas de l'oxygène atomique, la zone de transition entre oxydation active et oxydation passive est décalée dans le diagramme log $P=f(1 / T)$ vers les hautes températures pour une même pression partielle d'oxygène, ou pour une même température vers les basses pressions. Ceci a déjà été observé dans le cas de très basses pressions (de $10^{-2}$ à 10 pa) et à haute température (1750 à $2400 \mathrm{~K})$ par ROSNER /7/ sur du Sic pyrolytique. Dans notre cas, pour la temperature de $1850 \mathrm{~K}$, la pression partielle d'oxygène critique diminue de $3546 \mathrm{~Pa}$ dans le cas de $\mathrm{O}_{2}$ à $1013 \mathrm{~Pa}$ dans le cas de 0 soit une variation d'un facteur 3,5. La différence observée entre l'oxygène atomique et moléculaire penche fortement en faveur d'un contrôle cinétique de la réaction, contrairement à ce que prévoit la thermodynamique. Sur les photos de la figure 4, on observe une différence entre les états de surface. La photo (a) correspondant au point $C^{\prime}(0 ; 1676 \mathrm{~K}, 700 \mathrm{~Pa})$ présente une couche de silice fine sans bulles alors que la photo (c) correspondant au point $F^{\prime}(0,1806 \mathrm{~K}, 1000$ $\mathrm{Pa}$ comporte une couche de silice proche de celle de $C^{\prime}$ avec en plus la présence de bulles. La photo (b) correspondant au point $\mathrm{E}\left(\mathrm{O}_{2}, 1779 \mathrm{~K}\right.$, $2100 \mathrm{~Pa}$ ) présente une couche plus épaisse de silice où coexistent les phases cristallisée ( $\alpha$ cristobalite) et amorphe et où 1 'on observe aussi la présence de bulles.

\section{5 - CONCLUSION}

Nous avons mis au point un dispositif expérimental qui permet de tester des matériaux céramiques à haute température, basse pression et dans des conditions d'atmosphère moléculaire ou atomique grâce à un générateur de microondes. Après une étude thermodynamique prévisionnelle permettant de définir la zone de transition entre oxydation active et passive, des essais ont été effectués sur des échantillons de sic fritté, au foyer d'un four solaire à concentration de rayonnement dans deux atmosphères moléculaire et dissociée. En atmosphère moléculaire, les résultats 
experimentaux ont permis de confirmer la prévision thermodynamique. Par contre, en atmosphere atomique, un deplacement de la droite log $P_{02}^{\infty}=f(1 / T)$ représentant la transition a été observé pour de plus basses pressions, correspondant donc à une extension du domaine d'existence de la couche protectrice de silice. Ceci est interessant, en particulier en ce qui concerne les materiaux de bouclier thermique d'engins spatiaux, pour lesquels l'atmosphère dissociée environnante ne détruit pas la couche de protection formée même à basse pression. Dans la suite de ce travail, nous nous attacherons à mieux caractériser le plasma d'air généré (concentration d'oxygène atomique...), à étudier ${ }^{\prime}$ 'effet du débit de gaz vecteur, à poursuivre la détermination plus fine de la zone de transition en particulier dans le cas de l'oxygène atomique ainsi qu'à la caractérisation fine des états de surface par spectroscopie Auger.

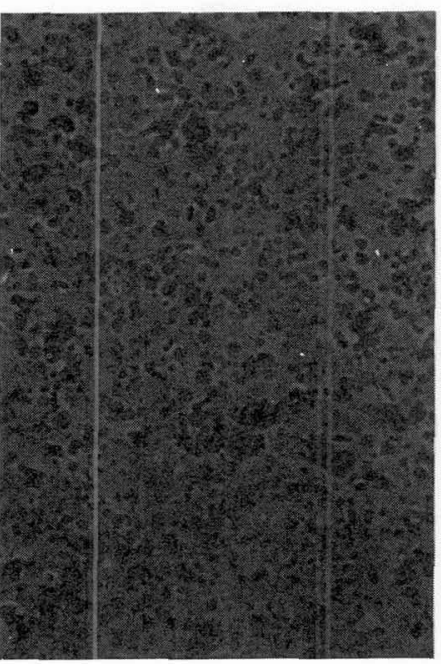

(a)

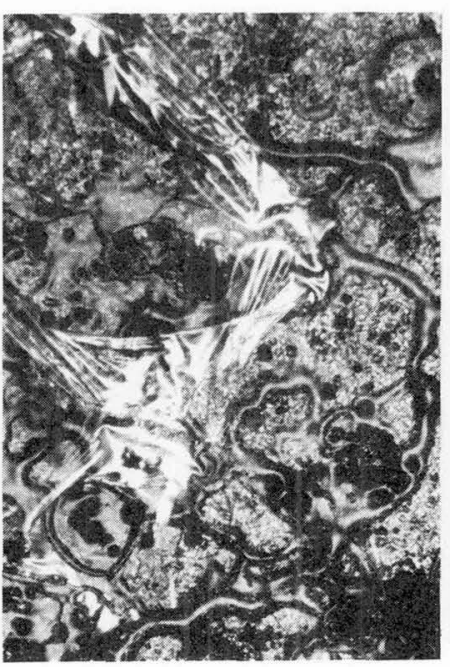

(b)

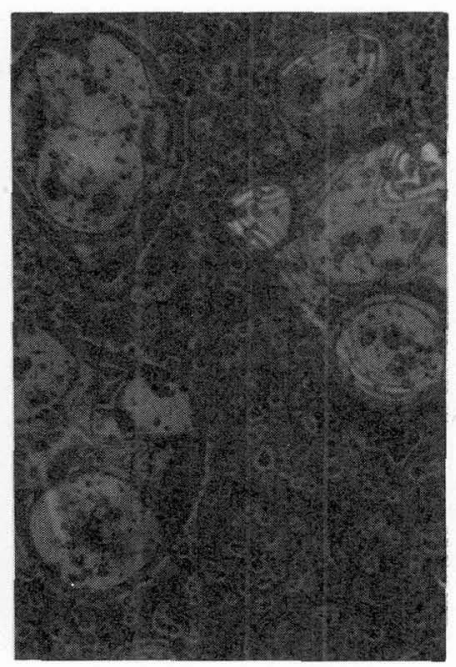

(c)

Figure 4: Photographies en microscopie optique des couches de silice : (a) échantillon $C^{\prime}$ (G X500); (b) échantillon $E$ (G X200) ; (c) échantillon $F^{\prime}$ (G $\times 500$ ).- Photographs of silica layers by optical microscopy : (a) sample $C^{\prime}(G \times 500)$; (b) sample E (G $\left.\times 200\right)$; (c) sample $F^{\prime}(G \times 500)$.

\section{REFERENCES}

/1/ TOULOUKIAN Y.S., De WITT D.P., Thermal radiative properties. Non metallic solids, 8 (1972) Plenum (N.Y.).

12/ WAGNER C., J. Appl. Phys., 29 (1958) 1295-97.

/3/ GULBRANSEN E.A., ANDREW K.F., BRASSART F.A., J. Electrochem. SOc., 113 (1966) 834-37.

14 SINGHAL S.C., Ceramurgia Int., $\underline{2}$ (1976) 123-30.

15/ JANAF, Thermochemical tables, J. Phys. Chem. Ref. Data, 14 (1985).

16/ DIAMY A.M., LEGRAND J.C., AL ANDARI J., BEN-AIM R.I., 9th Int. Symp. Plasma Chem., Pugnochiuso (Italy) 1 (1989) 591-96.

/7/ ROSNER D.E., ALLENDORF H.D., J. Phys. Chem., 74 (1970) 1829-39. 